pasteurisé. Ces défectuosités peuvent être dues à la destruction de la globuline et de l'albumine, car, comme Ch. Porcher [8] l'a montré, la consistance du coagulum produit par la présure est nettement influencée par la présence de protéines telles que l'albumine et la globuline, et ceci à un degré tel, que, dans les laits globulineux (femme, jument, chienne), l'action protectrice de ces substances empêche la formation d'un véritable caillé. La formation d'un caillé consistant, tel que celui qui se produit lorsque la globuline et l'albumine sont absentes, est cause d'un grand nombre de troubles digestifs chez les petits enfants et chez les jeunes animaux alimentés au lait de vache. La connaissance des variations naturelles de la composition des laits a donc, à ce point de vue, des applications importantes dans l'alimentation de tous les jeunes animaux.

(Traduction de R. N. Göransson.)

\title{
BIBLIOGRAPHIE.
}

[1] Asdell. Journ. Agr. Sc., 1925, 15.

[2] Asdell et Robinson. Journ. Physiol., 1926, 61.

[3] Boutflour. World's Dairy Congress, Londres, 1928. Proceedings.

[4] Hammond. La reproduction chez la vache, Cambridge, 1927.

[5] Magkenzie, Marshall et Hammond, Journ. Agr. Sc., 1911 à 1916, 4, 5, et 7.

[6] Orta-Jensen. World's Dairy Congress, Londres, 1928. Proceedings.

[7] Porcher. Compte rendu de l'Académie des Sciences, 1904, 138.

[8] Porcher. Le lait au point de vue colloïdal, Lyon, 1929.

[9] Sanders. Journ. Agr. Sc., 1927-28, 17 et 18.

[10] Woodman et Hammond. Journ, Agr. Sc, 1922, 12.

[11] Woodman et Hammond. Journ. Agr. Sc., 1923, 13.

\section{ÉTUDE DES CHAMPIGNONS TROUVÉS DANS LE LAIT LA CRÈME ET LE BEURRE}

$$
\text { par }
$$

\author{
M. GRIMES \\ Section de Bactériologie laitière
}

et

H. A. CUMMINS et V. C. E. KENNELLY

Section de Botanique

Université de Cork (Etat libre d'Irlande)

(Fin).

Chætomium bostrychoides Zopf [16] (P1. 22, fig. 14).

Cette forme, qui est très variable, fut trouvée une fois des périthéces apparaissant dans les cultures. Les périthéces sont gris acier, ovales, mais peuvent 
varier en passant par toutes les formes jusqu'à être globulaires, avec cirrhes (vrilles ?) noirs, droits ou recourbés. Les poils latéraux ne sont pas nombreux; couverts d'une croûte claire et uniformément cloisonnés et effilés ; brun olive à la base, près de $4 \mu$ de diamètre, et devenant de couleur plus claire vers les extrémités, fréquemment enroulées.

Les poils terminaux sont couverts d'une croûte et grossiers, brun sombre à noir, d'environ $4 \mu$ de diamètre, et généralement enroulés en spirale, irrégulièrement cloisonnés, et avec quelques rares ramifications cloisonnées et enroulées en spirale.

Les spores jeunes sont hyalines, verdâtres, avec un contenu granuleux; quand elles sont mûres, ovales et presque sphériques, quelquefois apiculées, ou arrondies aux deux extrémités, $4 \times 5 \mu$ dans notre type.

Cultures sur lait. — Solubilisation alcaline.

Tolérance à la chaleur. - Croissance à $60^{\circ} \mathrm{C}$. Pas de eroissance à $70^{\circ} \mathrm{C}$.

Tolérance au sel. - Légère croissance en solution de Czapek contenant $5 \%$ de $\mathrm{NaCl}$. Pas de croissance quand il y a $10 \%$ de $\mathrm{NaCl}$.

$p H$. - Peu ou pas de changement solution de Czapek contenant $5 \%$ de $\mathrm{NaCl}$.

Gélatine. - Liquéfaction partielle.

Production de diastase. - Faible.

\section{Phoma destructiva Flown.}

Le mycelium se développant sur lactose-agar nutritif forme un tapis épais, et est composé d'hyphes cloisonnés, ramifiés. Les cellules des hyphes aériens sont longs, 4 à 5 fois leur largeur, mais la longueur et le diamètre des cellules des hyphes submergés varient considérablement. Comparativement peu d'hyphes consistent en longues cellules étroites. La plus grande partie du mycelium est en grande majorité composée de cellules courtes, irrégulières, quelquefois presque complètement arrondies au point d'être séparées les unes des autres. Les pycnides sont rares. Elles ont des formes enfoncées, ouvertes par des ostioles, et sont généralement recouvertes par le mycelium aérien. Elles sont de couleur brune ou noire.

Les pyenides varient en dimensions de $60 \times 100-150-200 \mu$. La dimension moyenne des spores est de $3,5 \times 6-7 \mu$.

Quand on inocule une tomate, le fruit est presque complètement détruit en 21 jours. Les tissus devinrent tout à fait mous, des pycnides furent produites et le mycelium devint charbonneux z̀ la surface. Cette espèce n'a pas attaqué le chou, la laitue et la lavande.

Cultures sur lait. - Coagulation molle suivie par solubilisation.

Gelatine, - Liquéfaction marquée en 3 semaines.

Action diastasique. - Nulle ou très légère.

\section{Phoma hibernica Grimes.}

Le mycelium se développant sur lactose-agar nutritif est composé d'hyphes ramifiés, cloisonnés. Pycnides abondantes. Les pycnides sont enfoncées et ouvertes par des ostioles. Dimensions des pyenides variant de $60 \times 50-100$ - $200 \mu$. Les spores sont hyalines, unicellulaires, oblongues, arrondies grossièrement aux deux extrémités et fréquemment bigutullées. Les spores mesurent $4 \times 5-7 \mu$. Quelques-unes, rares, sont arrondies et mesurent $3 \mu$ de diamètre. Ensemencé sur chou, moutarde des champs, jacobée, pâquerette. 
tomate, sonehus, laitue, lavande, et trouvé non-parasitaire. Se développe sur milieux alimentaires réglés à un $p \mathrm{H}$ de 3,5 .

Cultures sur lait. - Bonne croissance en surface, réaction légèrement alcaline. Gélatine. - Moins de $5 \%$ de liquéfaction en 3 semaines.

Production de diastase. - Aucune.

Graphium penicillioides Corda 30 (Pl. 22, fig. 9, A, B et C).

Apparu sous forme de coussins duveteux de mycelium blanc; les conidies apparaissent sur des conidiophores ramifiés ou non (fig. 9, C); quelquefois, elles peuvent être produites par le mycelium sans conidiophore.

Les conidies sont un peu longues et de dimensions variables. Quelques hyphes épaissis apparaissent dans la colonie, et deviennent de couleur sombre ; ceux-ci portent aussi des conidies, et, plus tard, des fructifications du type graphium. La durée de la production varie considérablement; elle est plus lente sur agarlactose que sur agar Czapek ou agar Richard (fig. 9).

Les graphia (P1. 22, fig. 9, A et B) sont eourts, gros, à piliers eylindriques noirs, portant une tête blanc laiteux sphérique. Chacune naît de cellules renflées dans le mycelium, un cordon épais est formé et les hyphes qui le constituent peuvent être tordus, du sommet desquels se ramifient de petites conidies incolores. Le liquide dans lequel sont les spores est miscible à l'eau. Aucun périthéce n'est apparu dans nos cultures.

Cultures sur lait. - Forte croissance, solubilisation dù caillé, réaction acide.

Tolérance à la chaleur. - Légère croissance du type cladosporium quand il est conservé 3 semaines à $5^{\circ} \mathrm{C}$. Meilleure croissance à $8^{\circ} \mathrm{C}$. pendant le même temps.

Tolérance au sel. - Pas de croissance en solution de Czaper contenant $20 \%$ de $\mathrm{NaCl}$.

Gélatine. - Liquéfaction partielle en 10 jours.

Production de diastase. - Belle.

Coniosporium arundinis Corda ([5], vol. 8, p. 558) (Pl. 22, fig. 10, A et B; P1. 23, fig. 2).

Forme difficile à reconnaître, car la eroissance dans les milieux alimentaires est entièrement différente des formes trouvées sur les arbres; les spores caractéristiques avee bord hyalin l'identifient toutefois comme une espèce de Coniosporium.

Cloisonnement rampant, mycelium blane, avec spores vert olive, environ $6 \mu$. de long (voir P1 22, fig. 10, B). Les spores, qui sont cireulaires on do contours subanguleux, et lenticulées, sont portées aux sommets des conidiophores, chacune attachée par une petite excroissance, qui devient gélatineuse et disparaît, de sorte que les spores ont l'apparence d'être portées en têtes ou bouquets. Plus tard, tout le mycelium disparaît, et des taches noires sont visibles ultérieurement sous le mycelium; celles-ci peuvent perdre leur apparence arrondie et se souder, formant de longues raies (Pl. 23, fig. 2); plus tard, elles atteignent la surface supérieure du milieu. On voit alors que ce sont des masses de pores vert olive ayant jusqu'à $6,6 \mu$ de long.

Cultures sur lait. - Acide, coagulation suivie de solubilisation.

Tolérance à la chaieur. - Pas de croissance d̀ $70^{\circ} \mathrm{C}$. Légère croissance à $5^{\circ} \mathrm{C}$. Belle croissance à $8^{\circ} \mathrm{C}$. 
Tolérance au sel. - Belle croissance en solution de CzApEk contenant $12 \%$ de $\mathrm{NaCl}$. Pas de croissance en solution de Czapek contenant $15 \%$ de $\mathrm{NaCl}$.

$p H$. - Réaction non modifiée en solution de Czaper avec et sans addition de $\mathrm{NaCl}$.

Gélatine. - Liquéfaction partielle en 10 jours, "acervuli " brunâtres, transparents sur la surface du milieu et les eôtés du tube.

Production de diastase. - Bonne.

Alternaria spp. (P1. 23, fig. 3).

Plusieurs espèces furent trouvées. Dans une espèce, la tige et les spores sont grossières et de couleur brune, avec des becs incolores entre les spores en chainettes et de dimensions irrégulières. La forme appartient au groupe $A$. tenuis décrit par Mason, quoique les spores soient quelquefois ovales plutôt que obclavées.

Cultures sur lait. - Acide, coagulation suivie par lente solubilisation.

Tolérance à la chaleur. - Croissance à $70^{\circ} \mathrm{C}$. Pas de croissance à $75^{\circ} \mathrm{C}$.

Tolérance au sel. - Très légère eroissance en solution de CzApek contenant 15. \% de $\mathrm{NaCl}$. Pas de croissance en solution de CzapeK contenant $20 \%$ de $\mathrm{NaCl}$.

$p H$. - $p$ H du milieu ramené à 5,5 en solution de Czaper.

Gélatine. - Lente liquéfaction en 12 jours.

Production de diastase. - Faible.

Une espèce d'Alternaria fut trouvée se développant sur des planches de sapin importées pour la construction.

Torula Pers. (noir) (P1. 17, fig. 16).

Diverses espèces trouvées, mais n'ont pas pu être identifiées. Une espèce (Pl. 22, fig. 13) avait des spores $4 \mu \times 3 \mu$.

Sur une autre espèce envoyée à l'identification, on a trouvé que les chaînes typiques suggèrent en apparence les vieilles descriptions de Dematium hispidulum, mais seraient maintenant plutôt classées dans le même genre que le Torula, tel que le comprennent les mycologues.

Tolérance au sel. - Croissance en solution de Czapek contenant $5 \%$ et $10 \%$ de $\mathrm{NaCl}$. Pas de croissance en solution de Czaper contenant $15 \%$ de $\mathrm{NaCl}$.

Production de diastase. - Faible.

D'autres espèces variées de moisissures furent envoyées pour identification au Département impérial de Mycologie, à Kew. Quand l'identification fut reçue, les cultures originelles avaient été contaminées ou perdues, et aucun travail ultérieur ne fut exécuté. Ces espèces comprenaient :

Spicaria sp.

Pacilomyces sp. (probablement Eidamia catenulata Horne et William. son).

Gibberella moniliformis Wint, conidial.

Pilobolus crystallinus Tode.

\section{DISCUSSION.}

La présente recherche montre que beaucoup d'espèces différentes de moisissures peuvent se trouver dans le lait, la crème et le beurre. Leur présence dans le lait est probablement une suite de contamination, due aux conditions dans lesquelles les vaches sont 
logées, nourries et traites, et au nettoyage incomplet des ustensiles de laiterie. Des moisissures sont souvent présentes dans les produits alimentaires employés et les spores sont éparpillées dans l'air et ainsi dans le lait ; d'autres sont apportées par la poussière adhérant à l'animal, ou peuvent avoir leur origine dans le fumier. Les principales moisissures trouvées dans le lait par ordre de fréquence sont: le Penicillium, l'Oospora (Oidium) lactis, le Phoma, des espèces d'Aspergillus ; des espèces de Cladosporium (Hormodendrum) sont plus rarement trouvées ; d'autres espèces sont rarement trouvées, et quand on les trouve, il y en a généralement moins de 20 par centimètre cube.

Comme toutes les crèmes examinées avaient été pasteurisées et refroidies avant barattage, on n'a trouvé que peu de moisissures, généralement Oospora (Oidium) lactis, des espèces de Penicillium ou de Phoma, provenant de post-contamination, ou de pasteurisation insuffisante. Il est rare de trouver des espèces de Cladosporium (Hormodendrum). Quoique beaucoup de moisissures trouvées dans le beurre furent aussi trouvées dans le lait, on considère comme improbable que le lait ait été la cause de la contamination, car la crème est pasteurisée et refroidie avant barattage, quoique cela puisse arriver si la pasteurisation a été insuffisante. La température de pasteurisation dans les laiteries irlandaises varie de $85^{\circ} \mathrm{C}$. à $95^{\circ} \mathrm{C}$. et aucune moisissure ne survit à cette température. L'explication probable de leur présence dans le beurre est que ces types de champignons sont très répandus et pénètrent dans le beurre à un moment quelconque de la fabrication. Les laiteries sont généralement situées à la campagne et des spores fongiques des arbres, arbustes, herbes et récoltes sont apportées par les courants d'air. Elles sont, du reste, bâties à proximité d'une grande route et ceci augmente le risque de contamination du beurre par des spores fongiques apportées parl'air.

A la laiterie, les machines et les courroies en mouvement maintiennent une circulation d'air, et des spores de champignons se développant à l'humidité ou en des endroits négligés sont dispersées dans l'air et arrivent ainsi dans la crème ou le beurre. Comme l'air dans la laiterie est habituellement saturé d'humidité et que la température moyenne y est de $60^{\circ} \mathrm{F}$. $\left(15^{\circ} 5 \mathrm{C}\right.$.), ces conditions sont généralement favorables à la formation de spores. Si la ventilation à la laiterie est insuffisante, les moisissures se développeront sur les murs humides, les enduits de tuyauteries ou les poutres en bois, s'ils ne sont pas peints à intervalles réguliers. La erème, après pasteurisation, est exposée à la contamination par l'air, puisqu'elle coule habituellement sur un réfrigérant à l'eau ou à la saumure, ouvert, et est ensuite encore refroidie dans une cuve ouverte. Les 
tuyaux et pompes à crème peuvent aussi être des causes de contamination.

Si on fait du fromage au même endroit, il y a danger de contamination de la crème et du beurre par des spores, principalement d'espèces de Penicillium; puis quand la crème a été barattée, le beurre est exposé, tandis qu'on le transporte de la baratte aux caisses, et les caisses terminées sont laissées pendant des durées variables sur le sol ou sur un wagonnet avant que le couvercle ne soit cloué. Le bois, le parchemin et le sel sont considérés comme des facteurs négligeables dans la contamination, à moins qu'ils ne soient restés exposés à un endroit humide. L'eau employée pour le lavage du beurre peut aussi être une souree de contamination, car elle est souvent conservée dans une cuve non couverte, mais quoique beaucoup d'échantillons d'eau employée pour le lavage du beurre dans les laiteries aient été examinés, on les a presque toujours trouvés exempts de moisissures. La baratte est considérée comme étant la partie principale du matériel, à côté du nettoyage défectueux des tuyauteries et des pompes, cause des troubles dus aux moisissures à la laiterie. Il est difficile de la nettoyer, et presque impossible de la stériliser, car des crevasses et des fentes se développent graduellement dans le bois et les cylindres-lisseurs, dans lesquels se loge de la matière organique. Cette matière organique sert de nourriture aux microorganismes et, dans les conditions ordinaires de traitement des barattes, il n'y a pas pénétration suffisante de la chaleur dans ces crevasses et fentes pour tuer les microorganismes.

Tandis qu'on reconnaît que les chiffres de la tolérance à la chaleur et au sel ne valent que pour les conditions dans lesquelles ils ont été obtenus, il faut noter que les résultats obtenus montrent les différences qui existent entre les diverses espèces de champignons. L'Oidium lactis est détruit quand il est chauffé à $60^{\circ} \mathrm{C}$., tandis que quelques champignons ne sont pas détruits à $75^{\circ} \mathrm{C}$. La plupart des champignons ne se développent pas à $5^{\circ} \mathrm{C}$.; mais une espèce de Penicillium, Fusarium, Mucor corymbifer et Sporotrichum carnis se développent à cette température. Pratiquement, tous les champignons se développent dans la solution de CzAPEK contenant $10 \%$ de $\mathrm{NaCl}$; la plupart d'entre eux ne se développent pas quand l'addition de sel est de $15 \%$, et tous voient leur développement arrêté par l'addition de $20 \%$ de $\mathrm{NaCl}$. La conclusion de ce qui précède est que $2,3 \%$ de $\mathrm{NaCl}$, ou plus, sont nécessaires pour arrêter le développement de la croissance des champignons dans le beurre.

RÉSUMÉ.

Nous avons étudié pendant ces quatre dernières aṇnées des 
champignons isolés du lait, de la crème et du beurre. Les espèces suivantes furent isolées du lait :

Aspergillus glaucus groupe Sporotrichum carnis Brooks et

Thom

Aspergillus luteo-niger Lutz

Aspergillus terreus Thom

Aspergillus fumigatus Fres.

Penicillium spp.

Gliocladium spp. Corda

Mucor racemosus Fres.

Mucor corymbifer Cohn

Mucor mucedo Linne

Mucor alternans Van Tieghem

Thamnidium elegans Link

Pilobolus crystallinus Tode.

\section{Hansford}

Acrostalagmus cinnabarinus Corda

Botytris cinerea Pers.

Trichothecium roseum Link

Oospora (Oidium) lactis Fres.

Macrosporium sarcinula Berk

Stemphyllium botrysum Wallr.

Stysanus microsporus Sacc.

Cladosporium spp.

Fusarium spp.

Phoma hibernica

Alternaria spp.

Comme toute la crème examinée avait été pasteurisée, relativement peu de moisissures y furent trouvées; elles provenaient d'une post-contamination. Elles comprenaient :

Oospora (Oidium) lactis Fres. Penicillium spp.

Phoma hibernica

Cladosporium spp.

Les espèces suivantes furent isolées du beurre :

Aspergillus glaucus groupe

Thom

Aspergillus terreus Thom

Aspergillus Sydowi Bainier et

Sartory

Aspergillus fumigatus Fres.

Aspergillus luteo-niger Lutz

Penicillium brevi-compactum

Dierckx var.

Penicillium Johannioli Zaleski

Penicillium cyclopium West-

ling var.

Acrostalagmus cinnabarinus

Corda

Botrytis cinerea Pers.

Trichothecium roseum Link

Oospora (Oidium) lactis Fres.

Stemphyllium spp. Wallr. com-

prenant S. ericoctonum
Penicillium Sartoryi Thom var. Penicillium spinulosum Thom var.

Gliocladium spp. Corda

Pacilomyces spp. comprenant

Kennelly et Grimes.

Pacilomyces hibernicum

Mucor racemosus Fres.

Mucor corymbifer Cohn.

Sporotrichum carnis Brooks et Hansford

Trichoderma lignorum Tode

Chcetomium bostrychoides Zopf

Phoma hibernica Grimes

Phoma destructiva Plowr

Graphium penicillioides Corda

Coniosporium arundinis Corda

Alternaria spp.

Torula Pers. (noir) 
Stysanus microsporus Sacc. Cladosporium spp.

Fusarium spp. comprenant $F$. reticulatum Mont.
Sporendonema casei Desmaz. Spicaria spp.

Gibberella moniliformis Wint.

Nous désirons remercier de l'aide qu'elle nous a prêtée la Section de Laiterie du Ministère de l'Agriculture de l'Etat libre d'Irlande. Elle nous a envoyé des échantillons de beurre pour examen. Nous remercions aussi : le $\mathrm{D}^{\mathrm{r}}$ van $\mathrm{B}$. GILMour, qui nous a envoyé des espèces de moisissures isolées par lui du beurre pour identification ; le $\mathrm{D}^{\mathrm{r}}$ C. Тном, du Laboratoire de Microbiologie de la Section de Chimie du Ministère de l'Agriculture à Washington (Etats-Unis), qui a iden'tifié des cultures d'Aspergilli et de Penicilli, ainsi que le Dr E. J. Butler, C.I.E., F.R.S., F.L.S., Mr. E. W. Mason, M.A., M.Sc, Mr. S. F. AsнBy, B.Sc., et Mr. S. P. Wiltshire, B.A., B.Sc., du Bureau impérial de Mycologie, Kew, Surrey, Angleterre, pour l'aide qu'ils nous ont prêtée dans l'identification et la vérification. Nos sincères remerciements aussi à la Société royale de Dublin, Etat libre d'Irlande, pour nous avoir permis l'utilisation de clichés et autorisé la publication de littérature déjà publiée dans ses comptes rendus scientifiques [3] [7] [8].

\section{BIBLIOGRAPHIE.}

[1] С. Тном et M. В. Снивсн. The Aspergilli, 1926.

[2] C. Тном. The Penicillia, 1930.

[3] V. C. E. Kenneliy et M. Grimes. Precilomyces hibernicum. Sc. Proc., R. D. S.- vol. 19 (N. S.), no 44, septembre 1930.

[4] F. T. Brooks et C. G. HANSFond. Mould growth upon coldstore meat. Br. Myc. Soc., vol. 8, p. 113, 1923.

[5] Rabenhorst. Kryptogamen Flora, 1910.

[6] A. H. Chrvers. A Monograph of the genera Chætomiun et Arabricha. Mem. Torrey. Bot. Club.

[7] H. A. Cummins, V. C. S. Kennelly et M. Grimes, A study of fungi found in milk. Sc. Proc., R. D. S., vol. 19 (N. S.), n² 25, septembre 1929.

[8] M. Grimes, V. C. E. Kenneliy et H. A. Cummins. A study of fungi found in butter. Sc. Proc., R. D. S., vol. 19 (N. S.), no 47, octobre 1930.

[9] S. M. Libbert. Churn contamination and methods of control. National Butter Journal, p. 16, avril 1931.

[10] A. M. Brown. New South Wales butter quality. Agric. Gazette N.S. Wales, vol. 39, p. 843,1928 .

[11] H. MACY et W. B. Combs. Field studies of the sources of moulds in butter. Agricultural Experiment Station, Univ. of Minnesota, Bull, no 235, mai 1927. 
[12] H. Macy et G. M. Pulkrabek. Parchment paper as a source of mould infection in butter. Agricultural Experiment Station, Univ. of Minnesota, Bull., no 242, février 1928.

[13] H. Macy, W. B. Combs et H. B. Morrison. Preliminary studies of churn sanitation. Journal of Dairy Science, vol. 14, n⿳ 5 , p. 404, septembre 1931.

[14] B. D. MaC Callum. Some wood-staining fungi. Transactions British Mycological Society, vol. 7, p. 232, 1922.

[15] E. G. Hood et A. H. White. The cause and prevention of mould in Canadian pasteurised butter. Canada Dept. of Agriculture, Bull., no 48 (N. S.), 1925 .

[16] H. MACY. Some of the factors influencing the growth of moulds in butter. Agricultural Experiment Station, University of Minnesota, Technical Bulletin, no 64, décembre 1929.

[17] H. MACY. Quantitative changes in the micro-flora of butter during storage. Journal of Dairy Science, vol. 13, no 3, p. 266, 1930.

[18] J. C. Brown et F. W. Bouska. Prevention of mould in creameries. The Creamery Manager (Irish Free State), vol. 22, $n^{\circ}$ 12, p. 28, décembre 1931.

[19] M. A. Josuyn et W. V. Cruess. A comparative investigation of certain filmforming fungi. Hilgardia (University of California), vol. 4, n 9 , novembre 1929.

[20] G. L. A. Ruehle. Keeping qualities of butter. Agricultural Experiment Station, Michigan State College, Technical Bulletin, n 102 , janvier 1930.

[21] M. Grimes. A study of the action of certain bacteria, yeasts and moulds on the keeping quality of butter in cold storage. Journal of Dairy Science, vol. $6, n^{\circ} 5$, p. $427,1923$.

[22] Orta-Jensen. Dairy Bacteriology, p. 123, 1931.

[23] C. Тном et R. H. Shaw. Mouldiness in butter. Journal of Agricultural Research, vol. 3, no 4, p. 301, 1915 .

[24] G. P. Clinton. Fungi of mouldy unsalted butter. Agricultural Experiment Station, U.S.A., Bull., no 222, p. 400, août 1920.

[25] G. E. V. Morgan. Moulds in unsalted butter. The New Zealand Journal of Agriculture, vol, 39, $\mathrm{n}^{\mathrm{os}} \mathrm{1}, 2$ et $3,1929$.

[26] H. Macy, W. B. Combs et H. B. Morrison. Junior. The churn as a source of moulds in butter. Journal of Dairy Science, vol. 14, $\mathrm{n}^{\circ} 5$, p. 398, septembre 1931. 


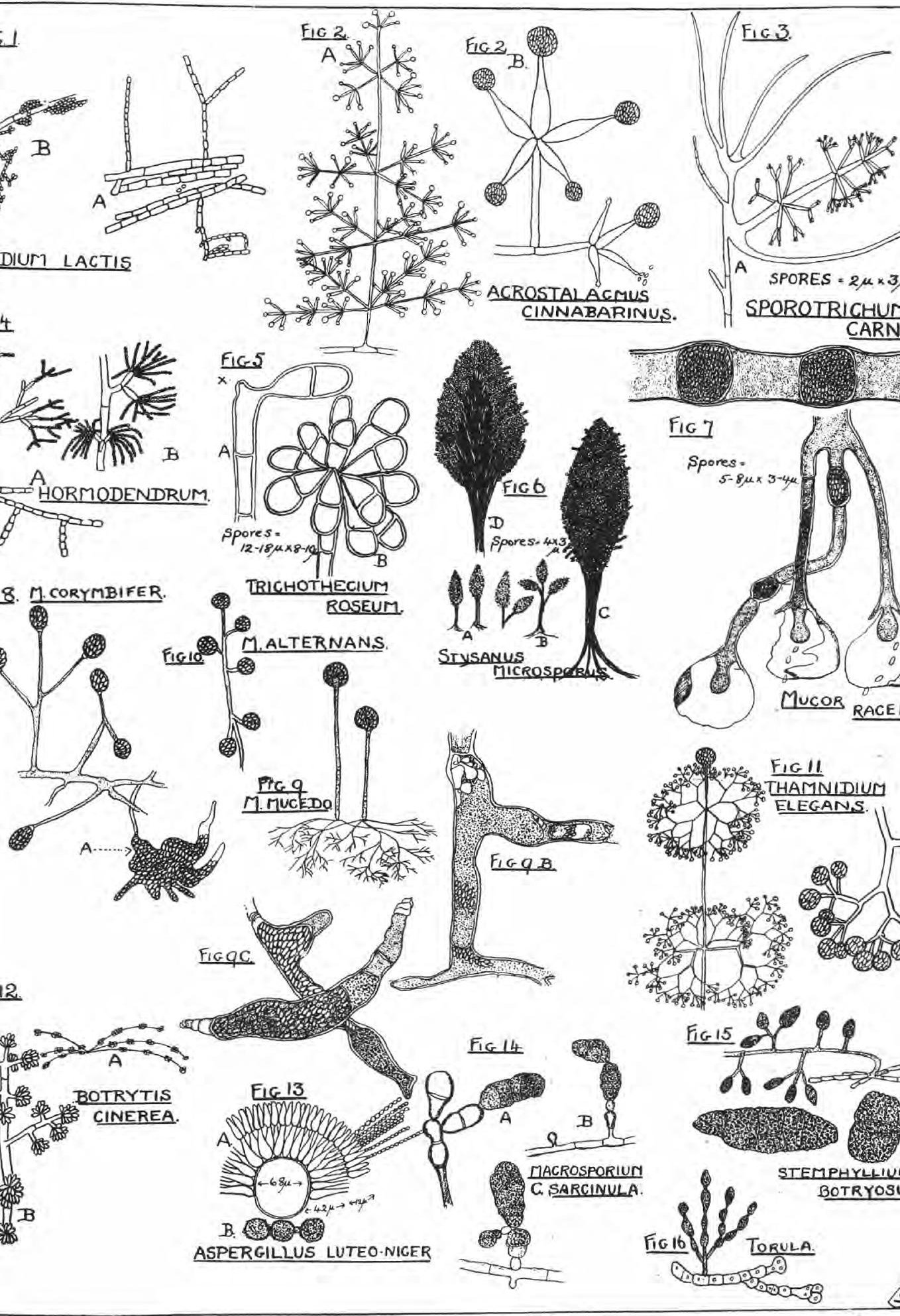


Fig.

Planche 17.

1. Oidium lactis Fresen.

$\mathrm{A}=$ mycelium ordinaire avec hyphe aérien.

$\mathrm{B}=$ état Torula.

2. Acrostalagmus cinnabarinus Corda.

$\mathrm{A}=$ petit grossissement, vue générale.

$\mathrm{B}=$ têtes conidiales, fort grossissement.

3. Sporotrichum carnis.

$\mathbf{A}=$ sporangiophores sous fort grossissement.

4. Hormodendrum.

$A=$ forme sur agar-dextrose.

$\mathrm{B}=$ forme sur agar Lemco $2 \%$.

5. Trichothecium roseum Link.

$A=$ conidie en formation en $\mathbf{X}$, une seconde étant formée.

$\mathrm{B}=$ tête de conidie.

6. Stysanus microsporus.

$\mathrm{A}=$ têtes solitaires.

$\mathrm{B}=$ ramification visible sur dextrose-agar.

$\mathrm{C}=$ tête simple grossie.

$\mathrm{D}=$ section à travers les coremies, montrant des hyphes fibreux d'où s'élancent des chaînes conidiales.

7. Mucor racemosus Fres.

$\mathrm{A}=$ chlamydospores en hyphe mycelien.

$\mathrm{B}=$ hyphe aérien avec sporanges et chlamydospores.

8. Mucor corymbifer Cohn.

$\mathrm{A}=$ structure ressemblant au chlamydospore dans le milieu alimentaire.

9. Mucor alternans.

10. Mucor mucedo Linné.

$\mathrm{B}$ et $\mathrm{C}=$ structures ressemblant à $8 \mathrm{~A}$.

11. Thamnidium elegans Link.

$\mathrm{A}=$ structure générale, petit grossissement.

$\mathbf{B}=$ sporanges secondaires fortement agrandis.

12. Botrytis cinerea Pers.

$\mathbf{A}=$ apparence générale, petit grossissement.

$\mathrm{B}=$ le même, agrandi.

13. Aspergillus luteo-niger Lutz.

$\mathrm{A}=$ section transversale par la tête, montrant la vésicule, le stérigmate et quelques chaînes de conidies.

$\mathrm{B}=$ spores sous fort grossissement. 

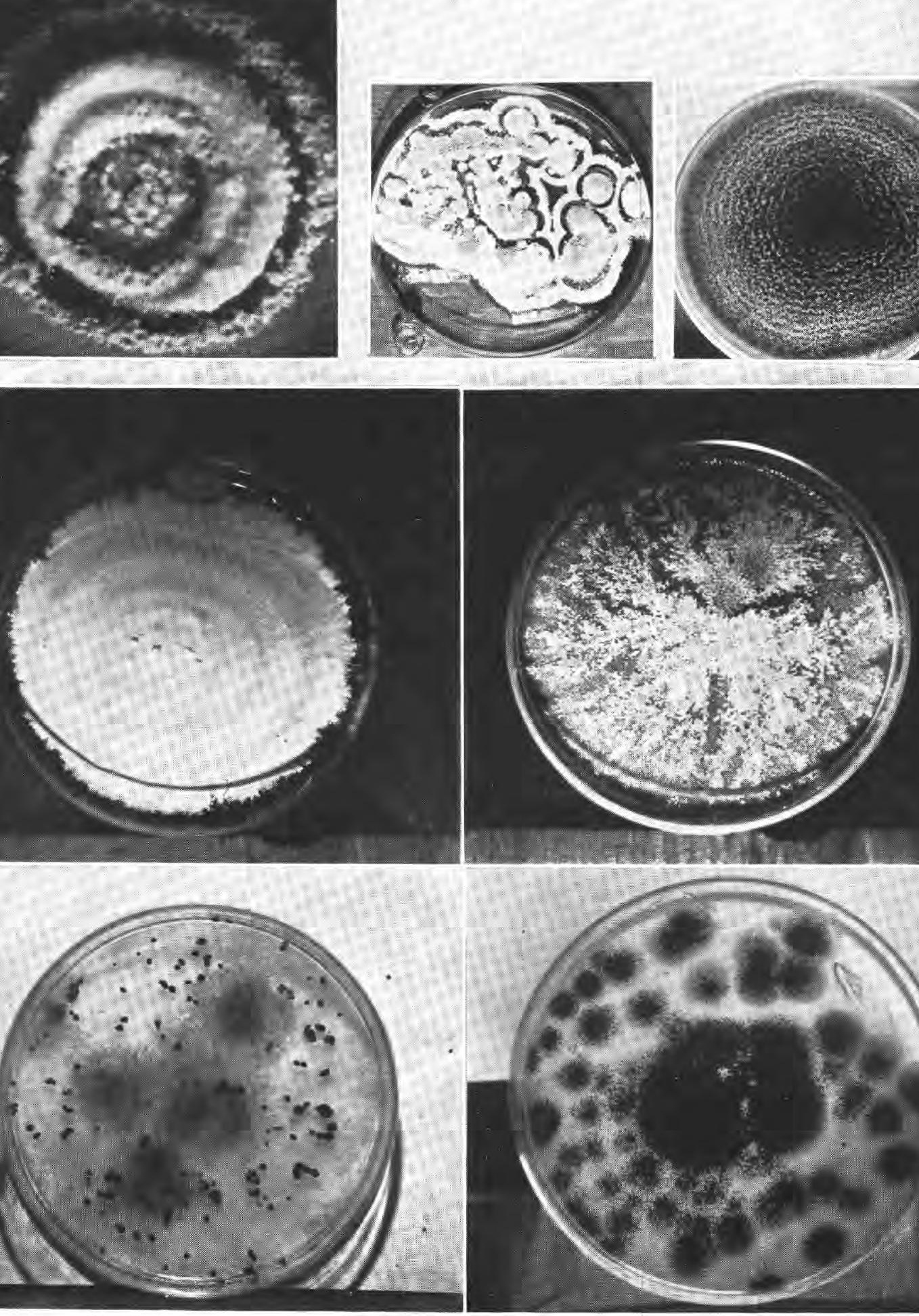
Fig.

Planche 17 (suite).

14. Macrosporium sarcinula.

Montrant des conidies en courtes chaînes, hyalines et lisses, quand jeunes.

- ultérieurement, sombres et verruqueuses.

$\mathrm{A}=$ sporangiophore.

15. Stemphyllium botryosum.

$\mathrm{A}=$ apparence générale, fort grossissement.

$\mathrm{B}, \mathrm{C}$ et $\mathrm{D}=$ conidies très agrandies.

16. Torula Pers.

Apparence générale, fort grossissement.

Fig.

Planche 18.

1. Sporotrichum carnis.

$\mathrm{A}=$ microphoto de colonie də $3 \mathrm{~m} / \mathrm{m} 12$ de diamètre.

$\mathrm{B}=$ culture sur agar-bacto-bœuf.

2. Trichothecium carnis Link.

$\mathrm{A}=$ croissance de colonie sur agar-pomme de terre.

$\mathbf{B}=$ croissance de colonie sur agar-bacto-bœuf.

3. Botrytis cinerea Pers.

Culture sur agar-pomme de terre, montrant des sclérotes noirs et des hyphes sombres portant des conidies.

4. Aspergillus luteo-niger Lutz.

Culture sur agar-dextrose.

5. Phoma hibernica Grimes.

Culture sur agar-dextrose.

Fig.

Planche 22.

1 A. Tête irrégulière de 1'Aspergillus glaucus var. montrant des ramifications d'où des têtes secondaires seront produites.

1 B. Stade ultérieur de têtes irrégulières analogues donnant l'apparence de têtes secondaires. 
Fig.

Planche 22 (suite).

1 C. Spores verruqueuses de ci-dessus.

2. Tête de A. fumigatus dessinée à l'échelle pour comparaison avec A. terreus, fig. 3 .

3. Tête en colonne de A. terreus dessinée à la même échelle que A. fumigatus, fig. 2 .

4. Dassin de la forme de $A$. fumigatus montrant la vésicule en forme de flacon et une rangée simple de stérigmates.

5. Dassin de la forme de $A$. terreus montrant une double rangée de stérigmates et de vésicules sphériques.

6. Tête de Penicillium brevi-compactum montrant l'apparence asymétrique caractéristique.

7 A. Tête genre Penicillium de Gliocladium sp.

7 B. Apparition de têtes couvertes de mucus sous fort grossissement.

8 A. Apparence sous fort grossissement de Trichoderma lignorum.

8 B. Ch́lamydospore intercalaire développé à basse température.

$8 \mathrm{C}$. Chlamydospore terminale de Trichoderma lignorum développé à basse température.

$9 \mathrm{~A}$ et $9 \mathrm{~B}$. Graphium penicillioides Corda; apparence générale de la fructification.

9 C. Stage cladosporium d,u Graphium penicillioides.

10 A. Spores de Coniosporium arundinis Corda.

10 B. Apparence de fructification jeune de Coniosporium arundinis Corda sous fort grossissement.

11 A. Spore de Fusarium, famille A.

11 B. Spore de Fusarium, famille C.

11 C. Stade microconidial de Fusarium spp.

11 D, E, F. Spores continues de Fusarium, famille A, cultivé en solution de Czapek.

11 G. Chaîne continue de microspores de Fusarium, famille B.

12 A. Apparence de Stemphyllium ericoctonum.

12 B. Cellules mycéliennes arrondies.

13. Torula sp.; aspect sous fort grossissement.

14. Chcetomiun bostrychoides Zopf.; aspect du perithèce.

Fig.

Planche 23.

1. - Microphotographie de têtes d'Aspergillus fumigatus.

2. - Cultura də Coniosporium arundinus moatrant l'apparence rayée due aux acervuli.

3. - Microphotographie d'une espèce d'Alternaria.

4. - Variété de Stemphyllium ericoctonum avec patites spores. 